\title{
Determination of an Unknown Coefficient in the Third Order Pseudoparabolic Equation with Non-Self-Adjoint Boundary Conditions
}

\author{
Yashar T. Mehraliyev and Gulshan Kh. Shafiyeva \\ Baku State University, 1148 Baku, Azerbaijan \\ Correspondence should be addressed to Yashar T. Mehraliyev; yashar_aze@mail.ru
}

Received 1 May 2014; Revised 11 July 2014; Accepted 22 July 2014; Published 13 August 2014

Academic Editor: Bo Han

Copyright (C) 2014 Y. T. Mehraliyev and G. Kh. Shafiyeva. This is an open access article distributed under the Creative Commons Attribution License, which permits unrestricted use, distribution, and reproduction in any medium, provided the original work is properly cited.

\begin{abstract}
The solvability of the inverse boundary problem with an unknown coefficient dependent on time for the third order pseudoparabolic equation with non-self-adjoint boundary conditions is investigated in the present paper. Here we have introduced the definition of the classical solution of the considered inverse boundary value problem, which is reduced to the system of integral equations by the Fourier method. At first, the existence and uniqueness of the solution of the obtaining system of integral equations is proved by the method of contraction mappings; then the existence and uniqueness of the classical solution of the stated problem is proved.
\end{abstract}

\section{Introduction}

Contemporary problems of natural sciences lead to the need for statement and investigation of the qualitative new problems. As an example we can consider a class of nonlocal problems for the partial differential equations. Researching such kind of problems aroused both theoretical interest and practical necessity and they are still studied actively today. The problems with both nonlocal boundary and initial conditions had previously been studied by many scientists. Classes of nonlocal problems with integral terms in boundary conditions are of great importance in the theory of heat conductivity, thermoelasticity, chemical engineering, underground water flow, population dynamics, and plasma physics.

The questions of solvability of the nonlocal problems with integral terms in boundary conditions had been studied by Samarskii [1]. Auxiliary information for investigation of the solution of such kind of problems can be found in [2-7]. Inverse problems with integral condition of override for pseudoparabolic type of equations had been studied in [8-10].

Existence and uniqueness of the solution of an inverse boundary value problem for the third order pseudoparabolic equation with the integral condition of override is proved in the present paper.

\section{Statement of the Problem and Reducing It to Equivalent}

Problem 1. Let us consider inverse boundary problem for the equation

$$
u_{t}(x, t)-b u_{t x x}(x, t)-a u_{x x}(x, t)=p(t) u(x, t)+f(x, t),
$$

in the domain $D_{T}=\{(x, t): 0 \leq x \leq 1,0 \leq t \leq T\}$ with initial condition

$$
u(x, 0)=\varphi(x) \quad(0 \leqslant x \leqslant 1),
$$

periodical condition

$$
u(0, t)=u(1, t) \quad(0 \leq t \leq T),
$$

Neumann boundary condition

$$
u_{x}(1, t)=0 \quad(0 \leqslant t \leqslant T),
$$

and the additional condition

$$
u\left(\frac{1}{2}, t\right)+\int_{0}^{1} u(x, t) d x=h(t) \quad(0 \leq t \leq T),
$$


where $a>0, b>0$ are the given numbers, $f(x, t), \varphi(x), h(t)$ are the given functions, and $u(x, t)$ and $p(t)$ are the unknown functions.

Definition 2. The classical solution of problems (1)-(5) is the pair $\{u(x, t), p(t)\}$ of the functions $u(x, t)$ and $p(t)$ possessing the following properties:

(a) $u(x, t)$ is continuous in $D_{T}$ together with all its derivatives contained in (1);

(b) $p(t)$ is continuous on $[0, T]$;

(c) all the conditions of (1)-(5) are satisfied in the ordinary sense.

The following lemma takes place.

Lemma 3. Suppose that $a>0, b>0, \varphi(x) \in C[0,1]$, $f(x, t) \in C\left(D_{T}\right), h(t) \in C^{1}[0, T], h(t) \neq 0(0 \leq t \leq T)$, and $\varphi(1 / 2)+\int_{0}^{1} \varphi(x) d x=h(0)$.

Then the problem of finding the classical solution of problem (1)-(5) is equivalent to the problem of defining the functions $u(x, t)$ and $p(t)$, possessing the properties (a) and $(b)$ of definition of the classical solution of problem (1)-(5), from relations (1)-(4), and

$$
\begin{aligned}
& h^{\prime}(t)+b\left(u_{t x}(0, t)+u_{t x x}\left(\frac{1}{2}, t\right)\right) \\
& +a\left(u_{x}(0, t)+u_{x x}\left(\frac{1}{2}, t\right)\right) \\
& \quad=p(t) h(t)+f\left(\frac{1}{2}, t\right)+\int_{0}^{1} f(x, t) d x
\end{aligned}
$$

Proof. Let $\{u(x, t), p(t)\}$ be a classical solution of problem (1)-(5). Differentiating (5) and taking into account that $h(t) \epsilon$ $C^{1}[0, T]$ we will get

$$
\frac{d}{d t}\left(u\left(\frac{1}{2}, t\right)+\int_{0}^{1} u(x, t) d x\right)=h^{\prime}(t) \quad(0 \leq t \leq T)
$$

From (1) we have

$$
\begin{aligned}
& \frac{d}{d t}\left(u\left(\frac{1}{2}, t\right)+\int_{0}^{1} u(x, t) d x\right) \\
& -b\left(u_{t x}(1, t)-u_{t x}(0, t)+u_{t x x}\left(\frac{1}{2}, t\right)\right) \\
& -a\left(u_{x}(1, t)-u_{x}(0, t)+u_{x x}\left(\frac{1}{2}, t\right)\right) \\
& \quad=f\left(\frac{1}{2}, t\right)+\int_{0}^{1} f(x, t) d x+p(t) \\
& \quad \times\left(u\left(\frac{1}{2}, t\right)+\int_{0}^{1} u(x, t) d x\right) \quad(0 \leq t \leq T) .
\end{aligned}
$$

Taking into account conditions (5) and (7) in (8), the fulfillment of condition (6) takes place.
Now suppose that $\{u(x, t), p(t)\}$ is a solution of problem (1)-(4), (6). Then from (6) and (8), taking into account (4), we find

$$
\begin{array}{r}
\frac{d}{d t}\left(u\left(\frac{1}{2}, t\right)+\int_{0}^{1} u(x, t) d x-h(t)\right) \\
-p(t)\left(u\left(\frac{1}{2}, t\right)+\int_{0}^{1} u(x, t) d x-h(t)\right)=0 \\
(0 \leq t \leq T) .
\end{array}
$$

Further, by (2) and $\varphi(1 / 2)+\int_{0}^{1} \varphi(x) d x=h(0)$, we obtain

$$
\begin{aligned}
& u\left(\frac{1}{2}, 0\right)+\int_{0}^{1} u(x, 0) d x-h(0) \\
& \quad=\varphi\left(\frac{1}{2}\right)+\int_{0}^{1} \varphi(x) d x-h(0)=0 .
\end{aligned}
$$

Since problems (9) and (10) has only a trivial solution, then $u(1 / 2, t)+\int_{0}^{1} u(x, t) d x-h(t)=0(0 \leq t \leq T)$; that is, the condition (5) is fulfilled.

\section{Auxiliary Facts}

Now, in order to investigate problem (1)-(4), (6) we introduce known facts. Consider the following spectral problem:

$$
\begin{gathered}
X^{\prime \prime}(x)+\mu X(x)=0 \quad(0 \leq x \leq 1), \\
X(0)=X(1), \quad X^{\prime}(1)=0,
\end{gathered}
$$

where $\mu$ is a parameter. Boundary value problem (11) is not self-adjoint [3]. The problem

$$
\begin{gathered}
Y^{\prime \prime}(x)+\mu Y(x)=0 \quad(0 \leq x \leq 1), \\
Y(0)=0, \quad Y^{\prime}(0)=Y^{\prime}(1)
\end{gathered}
$$

will be a conjugated problem.

Let us denote the system of eigen and adjoint functions of problem (11) in the following way:

$$
\begin{array}{r}
X_{0}(x)=2, \quad X_{2 k-1}=4 \cos \lambda_{k} x, \\
X_{2 k}(x)=4(1-x) \sin \lambda_{k} x \quad(k=1,2, \ldots),
\end{array}
$$

where

$$
\begin{gathered}
\lambda_{0}=0 \\
\lambda_{2 k-1}=\lambda_{2 k}=2 \pi k \quad(k=1,2, \ldots) .
\end{gathered}
$$

The system of function (13) forms a Riesz basis in the space $L_{2}(0,1)$.

Let us choose the system of eigen and adjoint functions of the conjugated problem as follows:

$$
\begin{aligned}
& Y_{0}(x)=x, \\
& Y_{2 k-1}=x \cos \lambda_{k} x, \quad Y_{2 k}(x)=4 \sin \lambda_{k} x \\
&(k=1,2, \ldots) .
\end{aligned}
$$


From this it follows that for systems (13) and (15) the biorthogonality condition in $L_{2}(0,1)$

$$
\left(X_{i}, Y_{j}\right)=\int_{0}^{1} X_{i}(x) Y_{j}(x) d x=\sigma_{i j}
$$

is satisfied. Here, $\sigma_{i j}$ is the Kronecker symbol.

Then the arbitrary function $g(x) \in L_{2}(0,1)$ is expanded in biorthogonal series:

$$
g(x)=\sum_{k=0}^{\infty} g_{k} X_{k}(x)
$$

and the following estimate is true:

$$
\frac{1}{2}\|g(x)\|_{L_{2(0,1)}}^{2} \leq \sum_{k=0}^{\infty} g_{k}^{2} \leq\|g(x)\|_{L_{2}(0,1)}^{2},
$$

where

$$
g_{k}=\int_{0}^{1} g(x) Y_{k}(x) d x .
$$

Under the assumptions

$$
\begin{array}{r}
g(x) \in C^{2 i-1}[0,1], \quad g^{(2 i)}(x) \in L_{2}(0,1), \\
g^{(2 s)}(0)=g^{(2 s)}(1), \quad g^{(2 s+1)}(1)=0 \\
(s=0,1, \ldots, i-1, i \geq 1),
\end{array}
$$

the following estimates hold:

$$
\begin{gathered}
\sum_{k=1}^{\infty}\left(\lambda_{k}^{2 i} g_{2 k}\right)^{2} \leq \frac{1}{2}\left\|g^{(2 i)}(x)\right\|_{L_{2}(0,1)}^{2} \\
\sum_{k=1}^{\infty}\left(\lambda_{k}^{2 i} g_{2 k-1}\right)^{2} \leq \frac{1}{2}\left\|x g^{(2 i)}(x)+2 i g^{(2 i-1)}(x)\right\|_{L_{2}(0,1)}^{2}
\end{gathered}
$$

Further, under the assumptions

$$
g(x) \in C[0,1], \quad g^{\prime}(x) \in L_{2}(0,1), \quad g(0)=g(1),
$$

the validity of the estimates is proved:

$$
\begin{gathered}
\sum_{k=1}^{\infty}\left(\lambda_{k} g_{2 k}\right)^{2} \leq \frac{1}{2}\left\|g^{\prime}(x)\right\|_{L_{2}(0,1)}^{2} \\
\sum_{k=1}^{\infty}\left(\lambda_{k} g_{2 k-1}\right)^{2} \leq \frac{1}{2}\left\|x g^{\prime}(x)+g(x)\right\|_{L_{2}(0,1)}^{2}
\end{gathered}
$$

Similarly, under the assumptions

$$
\begin{array}{r}
g(x) \in C^{2 i}[0,1], \quad g^{(2 i+1)}(x) \in L_{2}(0,1), \\
g^{(2 s)}(0)=g^{(2 s)}(1), \quad g^{(2 s-1)}(1)=0 \\
(s=0,1, \ldots, i, \quad i \geq 1),
\end{array}
$$

the estimations hold:

$$
\begin{gathered}
\sum_{k=1}^{\infty}\left(\lambda_{k}^{2 i+1} g_{2 k}\right)^{2} \leq \frac{1}{2}\left\|g^{(2 i+1)}(x)\right\|_{L_{2}(0,1)}^{2}, \\
\sum_{k=1}^{\infty}\left(\lambda_{k}^{2 i+1} g_{2 k-1}\right)^{2} \\
\leq \frac{1}{2}\left\|x g^{(2 i+1)}(x)+(2 i+1) g^{(2 i)}(x)\right\|_{L_{2}(0,1)}^{2}
\end{gathered}
$$

In order to investigate problem (1)-(4), (6), consider the following spaces.

(1) Denote by $B_{2, T}^{3}$ [11] the set of all the functions $u(x, t)$ of the form

$$
u(x, t)=\sum_{k=0}^{\infty} u_{k}(t) X_{k}(x)
$$

considered in $D_{T}$, where each of the functions $u_{k}(t) \quad(k=0,1, \ldots)$ is continuous on $[0, T]$ and

$$
\begin{aligned}
J_{T}(u) \equiv & \left\|u_{0}(t)\right\|_{C[0, T]} \\
& +\left(\sum_{k=1}^{\infty}\left(\lambda_{k}^{3}\left\|u_{2 k}(t)\right\|_{C[0, T]}\right)^{2}\right)^{1 / 2} \\
& +\left(\sum_{k=1}^{\infty}\left(\lambda_{k}^{3}\left\|u_{2 k-1}(t)\right\|_{C[0, T]}\right)^{2}\right)^{1 / 2}<+\infty .
\end{aligned}
$$

The norm in this set is determined as follows:

$$
\|u(x, t)\|_{B_{2, T}^{3}}=J_{T}(u) .
$$

(2) Denote by $E_{T}^{3}$ the space $B_{2, T}^{3} \times C[0, T]$ of the vector functions $z(x, t)=\{u(x, t), p(t)\}$ with the norm

$$
\|z(x, t)\|_{E_{T}^{3}}=\|u(x, t)\|_{B_{2, T}^{3}}+\|p(t)\|_{C[0, T]} .
$$

It is known that $B_{2, T}^{3}$ and $E_{T}^{3}$ are Banach spaces.

\section{Investigation of the Existence and Uniqueness of the Classical Solution of the Inverse Boundary Value Problem}

Since the system (13) is a Riesz basis in $L_{2}(0,1)$ and system (13), (15) is a system of biorthogonal functions in $L_{2}(0,1)$, it is obvious that for each solution $\{u(x, t), p(t)\}$ of problem (1)(4), (6) its first component $u(x, t)$ has the following form:

$$
u(x, t)=\sum_{k=0}^{\infty} u_{k}(t) X_{k}(x)
$$

where

$$
u_{k}(t)=\int_{0}^{1} u(x, t) Y_{k}(x) d x \quad(k=0,1, \ldots)
$$


Moreover, $X_{k}(x)$ and $Y_{k}(x)$ are defined by relations (13) and (15), respectively.

Then applying the formal scheme of the Fourier method, from (1) and (2), we have

$$
\begin{gathered}
u_{0}^{\prime}(t)=F_{0}(t ; u, p) \quad(0 \leq t \leq T), \\
\left(1+b \lambda_{k}^{2}\right) u_{2 k}^{\prime}(t)+a \lambda_{k}^{2} u_{2 k}(t)=F_{2 k}(t ; u, p) \\
(0 \leq t \leq T ; k=1,2, \ldots), \\
\left(1+b \lambda_{k}^{2}\right) u_{2 k-1}^{\prime}(t)+a \lambda_{k}^{2} u_{2 k-1}(t) \\
=F_{2 k-1}(t ; u, p)-2 \lambda_{k}\left(b u_{2 k}^{\prime}(t)+a u_{2 k}(t)\right) \\
(0 \leq t \leq T ; k=1,2, \ldots), \\
u_{k}(0)=\varphi_{k} \quad(k=0,1, \ldots),
\end{gathered}
$$

where

$$
\begin{gathered}
F_{k}(t ; u, p)=f_{k}(t)+p(t) u_{k}(t), \\
f_{k}(t)=\int_{0}^{1} f(x, t) Y_{k}(x) d x, \\
\varphi_{k}=\int_{0}^{1} \varphi(x) Y_{k}(x) d x \quad(k=0,1, \ldots) .
\end{gathered}
$$

Solving the problem (34)-(37), we find the following:

$$
\begin{aligned}
u_{0}(t)= & \varphi_{0}+\int_{0}^{t} F_{0}(\tau ; u, p) d \tau \quad(0 \leqslant t \leqslant T), \\
u_{2 k}(t)= & \varphi_{2 k} e^{-\left(a \lambda_{k}^{2} /\left(1+b \lambda_{k}^{2}\right)\right) t} \\
& +\frac{1}{1+b \lambda_{k}^{2}} \\
& \times \int_{0}^{t} F_{2 k}(\tau ; u, p) e^{-\left(a \lambda_{k}^{2} /\left(1+b \lambda_{k}^{2}\right)\right)(t-\tau)} d \tau \\
& (0 \leqslant t \leqslant T ; k=1,2, \ldots),
\end{aligned}
$$

$$
\begin{aligned}
u_{2 k-1}(t)= & \varphi_{2 k-1} e^{-\left(a \lambda_{k}^{2} /\left(1+b \lambda_{k}^{2}\right)\right) t} \\
& +\frac{1}{1+b \lambda_{k}^{2}} \\
& \times \int_{0}^{t} F_{2 k-1}(\tau ; u, p) e^{-\left(a \lambda_{k}^{2} /\left(1+b \lambda_{k}^{2}\right)\right)(t-\tau)} d \tau \\
& -\frac{2 a \lambda_{k} t}{\left(1+b \lambda_{k}^{2}\right)^{2}} \varphi_{2 k} e^{-\left(a \lambda_{k}^{2} /\left(1+b \lambda_{k}^{2}\right)\right) t}-\frac{2 a \lambda_{k}}{\left(1+b \lambda_{k}^{2}\right)^{3}} \\
& \times \int_{0}^{t}\left(\int_{0}^{\tau} F_{2 k}(\xi ; u, p) e^{-\left(a \lambda_{k}^{2} /\left(1+b \lambda_{k}^{2}\right)\right)(t-\xi)} d \xi\right) d \tau \\
& -\frac{2 b \lambda_{k}}{\left(1+b \lambda_{k}^{2}\right)^{2}} \\
& \times \int_{0}^{t} F_{2 k}(\tau ; u, p) e^{-\left(a \lambda_{k}^{2} /\left(1+b \lambda_{k}^{2}\right)\right)(t-\tau)} d \tau \\
& (0 \leq t \leq T ; \quad k=1,2, \ldots) .
\end{aligned}
$$

After substituting expressions $u_{k}(t)(k=0,1, \ldots)$ into (32) for determining the component $u(x, t)$ of the solution $\{u(x, t), p(t)\}$ of problem (1)-(4), (6) we get

$$
\begin{aligned}
& u(x, t) \\
& =\left(\varphi_{0}+\int_{0}^{t} F_{0}(\tau ; u, p) d \tau\right) X_{0}(x) \\
& +\sum_{k=1}^{\infty}\left(\varphi_{2 k} e^{-\left(a \lambda_{k}^{2} /\left(1+b \lambda_{k}^{2}\right)\right) t}\right. \\
& \left.+\frac{1}{1+b \lambda_{k}^{2}} \int_{0}^{t} F_{2 k}(\tau ; u, p) e^{-\left(a \lambda_{k}^{2} /\left(1+b \lambda_{k}^{2}\right)\right)(t-\tau)} d \tau\right) \\
& \times X_{2 k}(x) \\
& +\sum_{k=1}^{\infty}\left(\varphi_{2 k-1} e^{-\left(a \lambda_{k}^{2} /\left(1+b \lambda_{k}^{2}\right)\right) t}+\frac{1}{1+b \lambda_{k}^{2}}\right. \\
& \times \int_{0}^{t} F_{2 k-1}(\tau ; u, p) e^{-\left(a \lambda_{k}^{2} /\left(1+b \lambda_{k}^{2}\right)\right)(t-\tau)} d \tau \\
& -\frac{2 a \lambda_{k} t}{\left(1+b \lambda_{k}^{2}\right)^{2}} \varphi_{2 k} e^{-\left(a \lambda_{k}^{2} /\left(1+b \lambda_{k}^{2}\right)\right) t}-\frac{2 a \lambda_{k}}{\left(1+b \lambda_{k}^{2}\right)^{3}} \\
& \times \int_{0}^{t}\left(\int_{0}^{\tau} F_{2 k}(\xi ; u, p) e^{-\left(a \lambda_{k}^{2} /\left(1+b \lambda_{k}^{2}\right)\right)(t-\xi)} d \xi\right) d \tau \\
& -\frac{2 b \lambda_{k}}{\left(1+b \lambda_{k}^{2}\right)^{2}} \\
& \left.\times \int_{0}^{t} F_{2 k}(\tau ; u, p) e^{-\left(a \lambda_{k}^{2} /\left(1+b \lambda_{k}^{2}\right)\right)(t-\tau)} d \tau\right) \\
& \times X_{2 k-1(x)} .
\end{aligned}
$$

Now, taking into account (32), from (6), we get

$$
\begin{aligned}
& p(t) \\
& =h^{-1}(t)\left\{h^{\prime}(t)-f\left(\frac{1}{2}, t\right)-\int_{0}^{1} f(x, t) d x\right. \\
& \left.+4 \sum_{k=1}^{\infty}\left((-1)^{k} \lambda_{k}^{2}+\lambda_{k}\right)\left(b u_{2 k}^{\prime}(t)+a u_{2 k}(t)\right)\right\} .
\end{aligned}
$$

Further, from (35), taking into account (40), we have

$$
\begin{aligned}
& b u_{2 k}^{\prime}(t)+a u_{2 k}(t) \\
& =\frac{a}{1+b \lambda_{k}^{2}} u_{2 k}(t)+\frac{b}{1+b \lambda_{k}^{2}} F_{2 k}(t ; u, p) \\
& =\frac{a}{1+b \lambda_{k}^{2}}\left(\varphi_{2 k} e^{-\left(a \lambda_{k}^{2} /\left(1+b \lambda_{k}^{2}\right)\right) t}+\frac{1}{1+b \lambda_{k}^{2}}\right.
\end{aligned}
$$




$$
\begin{aligned}
& \left.\times \int_{0}^{t} F_{2 k}(\tau ; u, p) e^{-\left(a \lambda_{k}^{2} /\left(1+b \lambda_{k}^{2}\right)\right)(t-\tau)} d \tau\right) \\
& +\frac{b}{1+b \lambda_{k}^{2}} F_{2 k}(t ; u, p) .
\end{aligned}
$$

To find the second component $p(t)$ of the solution $\{u(x, t), p(t)\}$ of problem (1)-(4), (6) after substituting expression (44) into (43), we get

$$
\begin{aligned}
p(t)=h^{-1}(t)\left\{h^{\prime}(t)-f\left(\frac{1}{2}, t\right)-\int_{0}^{1} f(x, t) d x\right. \\
+4 \sum_{k=1}^{\infty} \frac{(-1)^{k} \lambda_{k}^{2}+\lambda_{k}}{1+b \lambda_{k}^{2}} \\
\times\left[a \left(\varphi_{2 k} e^{-\left(a \lambda_{k}^{2} /\left(1+b \lambda_{k}^{2}\right)\right) t}+\frac{1}{1+b \lambda_{k}^{2}}\right.\right. \\
\left.\quad \times \int_{0}^{t} F_{2 k}(\tau ; u, p) e^{-\left(a \lambda_{k}^{2} /\left(1+b \lambda_{k}^{2}\right)\right)(t-\tau)} d \tau\right) \\
\left.\left.+b F_{2 k}(t ; u, p)\right]\right\} .
\end{aligned}
$$

Thus, the solution of problem (1)-(4), (6) is reduced to the solution of system (42) and (45) with respect to the unknown functions $u(x, t)$ and $p(t)$.

Now, proceeding from the definition of the classical solution of problem (1)-(4), (6) the following lemma is proved.

Lemma 4. If $\{u(x, t), p(t)\}$ is any solution of problem (1)-(4), (6), then the function

$$
u_{k}(t)=\int_{0}^{1} u(x, t) Y_{k}(x) d x \quad(k=0,1, \ldots)
$$

satisfies the system (39)-(41) in $[0, T]$.

Remark 5. From Lemma 4 it follows that, to prove the uniqueness of the solution of problem (1)-(4), (6), it suffices to prove the uniqueness of the solution of system (42), (45).

Now, in the space $E_{T}^{3}$ consider the operator

$$
\Phi(u, p)=\left\{\Phi_{1}(u, p), \Phi_{2}(u, p)\right\},
$$

where

$$
\begin{aligned}
& \Phi_{1}(u, p)= \widetilde{u}(x, t) \equiv \widetilde{u}_{0}(t) X_{0}(x) \\
&+\sum_{k=1}^{\infty} \widetilde{u}_{2 k}(t) X_{2 k}(x) \\
&+\sum_{k=1}^{\infty} \widetilde{u}_{2 k-1}(t) X_{2 k-1}(x), \\
& \Phi_{2}(u, p)=\tilde{p}(t),
\end{aligned}
$$

and $\widetilde{u}_{0}(t), \widetilde{u}_{2 k}(t), \widetilde{u}_{2 k-1}(t)$, and $\widetilde{p}(t)$ equal the right sides of (39), (40), (41), and (45), respectively.

It is easy to see that

$$
\begin{gathered}
b \lambda_{k}^{2}<1+b \lambda_{k}^{2}<(1+b) \lambda_{k}^{2}, \\
\frac{1}{(1+b) \lambda_{k}^{2}}<\frac{1}{1+b \lambda_{k}^{2}}<\frac{1}{b \lambda_{k}^{2}} .
\end{gathered}
$$

By means of simple transformations we find

$$
\begin{aligned}
& \left\|\widetilde{u}_{0}(t)\right\|_{C[0, T]} \\
& <\left|\varphi_{0}\right|+\sqrt{T}\left(\int_{0}^{T}\left|f_{0}(\tau)\right|^{2} d \tau\right)^{1 / 2} \\
& +T\|p(t)\|_{C[0, T]}\left\|u_{0}(t)\right\|_{C[0, T]}, \\
& \left(\sum_{k=1}^{\infty}\left(\lambda_{k}^{3}\left\|\tilde{u}_{2 k}(t)\right\|_{C[0, T]}\right)^{2}\right)^{1 / 2} \\
& \leq \sqrt{3}\left(\sum_{k=1}^{\infty}\left(\lambda_{k}^{3}\left|\varphi_{2 k}\right|\right)^{2}\right)^{1 / 2} \\
& +\frac{\sqrt{3 T}}{b}\left(\int_{0}^{T} \sum_{k=1}^{\infty}\left(\lambda_{k}\left|f_{2 k}(\tau)\right|\right)^{2} d \tau\right)^{1 / 2} \\
& +\frac{\sqrt{3}}{b} T\|p(t)\|_{C[0, T]} \\
& \times\left(\sum_{k=1}^{\infty}\left(\lambda_{k}^{3}\left\|u_{2 k}(t)\right\|_{C[0, T]}\right)^{2}\right)^{1 / 2}, \\
& \left(\sum_{k=1}^{\infty}\left(\lambda_{k}^{3}\left\|\tilde{u}_{2 k-1}(t)\right\|_{C[0, T]}\right)^{2}\right)^{1 / 2} \\
& \leq 2 \sqrt{2}\left(\sum_{k=1}^{\infty}\left(\lambda_{k}^{3}\left|\varphi_{2 k-1}\right|\right)^{2}\right)^{1 / 2} \\
& +\frac{2 \sqrt{2 T}}{b}\left(\int_{0}^{T} \sum_{k=1}^{\infty}\left(\lambda_{k}\left|f_{2 k-1}(\tau)\right|\right)^{2} d \tau\right)^{1 / 2} \\
& +\frac{2 \sqrt{2}}{b} T\|p(t)\|_{C[0, T]} \\
& \times\left(\sum_{k=1}^{\infty}\left(\lambda_{k}^{3}\left\|u_{2 k-1}(t)\right\|_{C[0, T]}\right)^{2}\right)^{1 / 2} \\
& +\frac{4 \sqrt{2} a}{b^{2}} T\left(\sum_{k=1}^{\infty}\left(\lambda_{k}^{3}\left|\varphi_{2 k}\right|\right)^{2}\right)^{1 / 2}+4 \sqrt{2}\left(\frac{1}{b}+\frac{a}{b^{3}} T\right) \sqrt{T} \\
& \times\left(\int_{0}^{T} \sum_{k=1}^{\infty}\left(\lambda_{k}\left|f_{2 k}(\tau)\right|\right)^{2} d \tau\right)^{1 / 2}
\end{aligned}
$$




$$
\begin{aligned}
&+ 4 \sqrt{2}\left(\frac{1}{b}+\frac{a}{b^{3}} T\right) T\|p(t)\|_{C[0, T]} \\
& \times\left(\sum_{k=1}^{\infty}\left(\lambda_{k}^{3}\left\|u_{2 k-1}(t)\right\|_{C[0, T]}\right)^{2}\right)^{1 / 2} \\
&\|\tilde{p}(t)\|_{C[0, T]} \leq\left\|h^{-1}(t)\right\|_{C[0, T]} \\
& \times\left\{\left\|h^{\prime}(t)-f\left(\frac{1}{2}, t\right)-\int_{0}^{1} f(x, t) d x\right\|_{C[0, T]}\right. \\
&+\frac{4}{\sqrt{6} b}\left[a\left(\sum_{k=1}^{\infty}\left(\lambda_{k}^{3}\left|\varphi_{2 k}\right|\right)^{2}\right)^{1 / 2}\right. \\
&+\frac{a}{b} \sqrt{T}\left(\int_{0}^{T} \sum_{k=1}^{\infty}\left(\lambda_{k}\left|f_{2 k}(\tau)\right|\right)^{2} d \tau\right)^{1 / 2} \\
&+b\left(\sum_{k=1}^{\infty}\left(\lambda_{k}\left\|f_{2 k}(t)\right\|_{C[0, T]}\right)^{2}\right)^{1 / 2} \\
&+\left(\frac{a}{b} T+b\right)\|p(t)\|_{C[0, T]} \\
&\left.\left.\times\left(\sum_{k=1}^{\infty}\left(\lambda_{k}^{3}\left\|u_{2 k}(t)\right\|_{C[0, T]}\right)^{2}\right)^{1 / 2}\right]\right\}
\end{aligned}
$$

Suppose that the data of problem (1)-(4), (6) satisfy the following conditions:

(1) $\varphi(x) \in C^{2}[0,1], \varphi^{\prime \prime \prime}(x) \in L_{2}(0,1), \varphi(0)=\varphi(1)$, $\varphi^{\prime}(1)=0, \varphi^{\prime \prime}(0)=\varphi^{\prime \prime}(1)$;

(2) $f(x, t) \in C\left(D_{T}\right), f_{x}(x, t) \in L_{2}\left(D_{T}\right), f(0, t)=$ $f(1, t)(0 \leqslant t \leqslant T)$;

(3) $a>0, b>0, h(t) \in C^{1}[0, T], h(t) \neq 0(0 \leqslant t \leqslant T)$.

Then taking into account (21)-(27), from (50), we get

$$
\begin{aligned}
& \|\widetilde{u}(x, t)\|_{B_{2, T}^{3}}+\|\widetilde{p}(t)\|_{C[0, T]} \\
& \quad \leq A(T)+B(T)\|p(t)\|_{C[0, T]}\|u(x, t)\|_{B_{2, T}^{3}},
\end{aligned}
$$

where

$A(T)$

$$
\begin{aligned}
= & \frac{1}{3}\|\varphi(x)\|_{L_{2}(0,1)}+\frac{1}{3} \sqrt{T}\|f(x, t)\|_{L_{2}\left(D_{T}\right)} \\
& +\left\|h^{-1}(t)\right\|_{C[0, T]}\left\|h^{\prime}(t)-f\left(\frac{1}{2}, t\right)-\int_{0}^{1} f(x, t) d x\right\|_{C[0, T]} \\
& +\left(\frac{\sqrt{6}}{2}+\frac{4 a}{b^{2}} T+\frac{\sqrt{3} a}{3 b}\left\|h^{-1}(t)\right\|_{C[0, T]}\right)\left\|\varphi^{\prime \prime \prime}(x)\right\|_{L_{2}(0,1)}
\end{aligned}
$$

$$
\begin{aligned}
& +\left(\frac{\sqrt{6 T}}{2 b}+4\left(\frac{1}{b}+\frac{a}{b^{3}} T\right) \sqrt{T}+\frac{\sqrt{3 T} a}{3 b^{2}}\left\|h^{-1}(t)\right\|_{C[0, T]}\right) \\
& \times\left\|f_{x}(x, t)\right\|_{L_{2}\left(D_{T}\right)} \\
+ & 2\left\|x \varphi^{\prime \prime \prime}(x)+3 \varphi^{\prime \prime}(x)\right\|_{L_{2}(0,1)}+\frac{\sqrt{3}}{3}\left\|h^{-1}(t)\right\|_{C[0, T]} \\
& \times\|\| f_{x}(x, t)\left\|_{C[0, T]}\right\|_{L_{2}(0,1)}, \\
B(T)= & \left(1+\frac{1}{b}(2 \sqrt{2}+\sqrt{3})+\frac{4 \sqrt{2}}{b}\left(1+\frac{a}{b^{2}} T\right)\right) T \\
& +\left(\frac{a}{b} T+b\right)\left\|h^{-1}(t)\right\|_{C[0, T]} .
\end{aligned}
$$

So, the following theorem can be proved.

Theorem 6. Let conditions (1)-(3) be satisfied, and

$$
(A(T)+2)^{2} B(T)<1 .
$$

Then problem (1)-(4), (6) has a unique solution in the ball $K=K_{R}\left(\|z\|_{E_{T}^{3}} \leq R=A(T)+2\right)$ of the space $E_{T}^{3}$.

Remark 7. Inequality (53) is satisfied for sufficiently small values at $T+\left\|h^{-1}(t)\right\|_{C[0, T]}$.

Proof. Consider the equation

$$
z=\Phi z
$$

in the space $E_{T}^{3}$, where $z=\{u, p\}$ and the components $\Phi_{i}(u, p)(i=1,2)$ of the operator $\Phi(u, p)$ are defined from the right sides of (42), (45). Consider the operator $\Phi(u, p)$ in the ball $K=K_{R}$ from $E_{T}^{3}$.

Similar to (51), for any $z, z_{1}, z_{2} \in K_{R}$ the following estimates hold:

$$
\begin{aligned}
& \|\Phi z\|_{E_{T}^{3}} \leq A(T)+B(T)\|p(t)\|_{C[0, T]}\|u(x, t)\|_{B_{2, T}^{3}}, \\
& \left\|\Phi z_{1}-\Phi z_{2}\right\|_{E_{T}^{3}} \leq B(T) R\left(\left\|p_{1}(t)-p_{2}(t)\right\|_{C[0, T]}\right. \\
& \left.+\left\|u_{1}(x, t)-u_{2}(x, t)\right\|_{B_{2, T}^{3}}\right) .
\end{aligned}
$$

Then taking into account (53) in (55), it follows that the operator $\Phi$ acts in the ball $K=K_{R}$ and is contractive. Therefore, in the ball $K=K_{R}$ the operator $\Phi$ has a unique fixed point $\{u, p\}$ that is a unique solution of (54) in the ball $K=K_{R}$; that is, it is a unique solution of system (42), (45) in the ball $K=K_{R}$.

The function $u(x, t)$ as an element of the space $B_{2, T}^{3}$ is continuous and has continuous derivatives $u_{x}(x, t)$ and $u_{x x}(x, t)$ in $D_{T}$. 
From (35), (36) it is easy to see that

$$
\begin{aligned}
& \left(\sum_{k=1}^{\infty}\left(\lambda_{k}^{3}\left\|u_{2 k}^{\prime}(t)\right\|_{C[0, T]}\right)^{2}\right)^{1 / 2} \\
& \leq \frac{\sqrt{2} a}{b}\left(\sum_{k=1}^{\infty}\left(\lambda_{k}^{3}\left\|u_{2 k}(t)\right\|_{C[0, T]}\right)^{2}\right)^{1 / 2} \\
& +\frac{1}{b}\|\| f_{x}(x, t)+p(t) u_{x}(x, t)\left\|_{C[0, T]}\right\|_{L_{2}(0,1)}, \\
& \left(\sum_{k=1}^{\infty}\left(\lambda_{k}^{3}\left\|u_{2 k-1}^{\prime}(t)\right\|_{C[0, T]}\right)^{2}\right)^{1 / 2} \\
& \leq \frac{\sqrt{5} a}{b}\left(\sum_{k=1}^{\infty}\left(\lambda_{k}^{3}\left\|u_{2 k}(t)\right\|_{C[0, T]}\right)^{2}\right)^{1 / 2}+\frac{\sqrt{10}}{2 b} \\
& \times\|\| x f_{x}(x, t)+f(x, t)+p(t) u_{x}(x, t) x \\
& +p(t) u(x, t)\left\|_{C[0, T]}\right\|_{L_{2}(0,1)} \\
& +2 \sqrt{5}\left(\sum_{k=1}^{\infty}\left(\lambda_{k}^{3}\left\|u_{2 k}^{\prime}(t)\right\|_{C[0, T]}\right)^{2}\right)^{1 / 2} \\
& +\frac{2 \sqrt{5} a}{b}\left(\sum_{k=1}^{\infty}\left(\lambda_{k}^{3}\left\|u_{2 k}(t)\right\|_{C[0, T]}\right)^{2}\right)^{1 / 2} .
\end{aligned}
$$

From the last relation it is obvious that $u_{t}(x, t), u_{t x}(x, t)$, and $u_{t x x}(x, t)$ are continuous in $D_{T}$.

It is easy to verify that (1) and conditions (2), (3), (4), and (6) are satisfied in the ordinary sense.

Consequently, $\{u(x, t), p(t)\}$ is a solution of problem (1)(4), (6) and by Lemma 4 this solution is unique in the ball $K=K_{R}$. The theorem is proved.

By means of Lemma 3 the uniqueness of the solution of the initial problem (1)-(5) follows from Theorem 6 and the following theorem takes place.

Theorem 8. Suppose that all the conditions of Theorem 6 are satisfied and

$$
\varphi\left(\frac{1}{2}\right)+\int_{0}^{1} \varphi(x) d x=h(0) .
$$

Then problem (1)-(5) has a unique classical solution in the ball $K=K_{R}\left(\|z\|_{E_{T}^{3}} \leqslant R=A(T)+2\right)$ of the space $E_{T}^{3}$.

\section{Conflict of Interests}

The authors declare that there is no conflict of interests regarding the publication of this paper.

\section{References}

[1] A. A. Samarskii, "On some problems of the theory of differential equations," Differentsial'nye Uravneniya, vol. 16, no. 11, pp. 19251935, 1980.
[2] J. R. Cannon, "The solution of the heat equation subject to the specification of energy," Quarterly of Applied Mathematics, vol. 21, no. 3, pp. 155-160, 1963.

[3] N. I. Ionkin, "The solution of a certain boundary value problem of the theory of heat conduction with a nonclassical boundary condition," Differentsial'nye Uravneniya, vol. 13, no. 2, pp. 294304, 381, 1977.

[4] Y. T. Mehraliyev and G. K. Shafiyeva, "Inverse boundary value problem for the pseudoparabolic equation of the third order with periodic and integral conditions," Applied Mathematical Sciences, vol. 8, no. 23, pp. 1145-1155, 2014.

[5] Y. T. Mehraliyev and G. K. Shafiyeva, "On an inverse boundary value problem for the pseudoparabolic equation of the third order with integral condition of the first kind," Ukrainian Mathematical Bulletin, vol. 11, no. 2, pp. 240-249, 2014.

[6] A. I. Kozhanov and L. S. Pul'kina, "On the solvability of boundary value problems with a nonlocal boundary condition of integral form for multidimensional hyperbolic equations," Differential Equations, vol. 42, no. 9, pp. 1233-1246, 2006.

[7] D. G. Gordeziani and G. A. Avalishvili, "On the constructing of solutions of the nonlocal initial boundary value problems for one-dimensional medium oscillation equations," Matematicheskoe Modelirovanie, vol. 12, no. 1, pp. 94-103, 2000.

[8] F. Kanca, "Inverse coefficient problem of the parabolic equation with periodic boundary and integral overdetermination conditions," Abstract and Applied Analysis, vol. 2013, Article ID 659804, 7 pages, 2013.

[9] A. I. Prilepko and D. S. Tkachenko, "Properties of solutions of a parabolic equation and the uniqueness of the solution of the inverse source problem with integral overdetermination," Zhurnal Vychislitel'noi Matematiki i Matematicheskoi Fiziki, vol. 43, no. 4, pp. 562-570, 2003.

[10] V. L. Kamynin, "On an inverse problem of determining the right-hand side of a parabolic equation with the integral overdetermination condition," Matematicheskie Zametki, vol. 77, no. 4, pp. 522-534, 2005.

[11] K. I. Khudaverdiyev and A. A. Veliyev, Investigation of OneDimensional Mixed Problem for a Class of Pseudohyperbolic Equations of Third Order with Nonlinear Operator Right Side, Chashyoglu, Baku, Azerbaijan, 2010. 


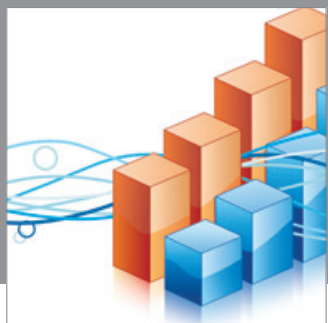

Advances in

Operations Research

mansans

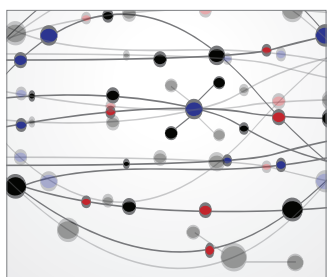

The Scientific World Journal
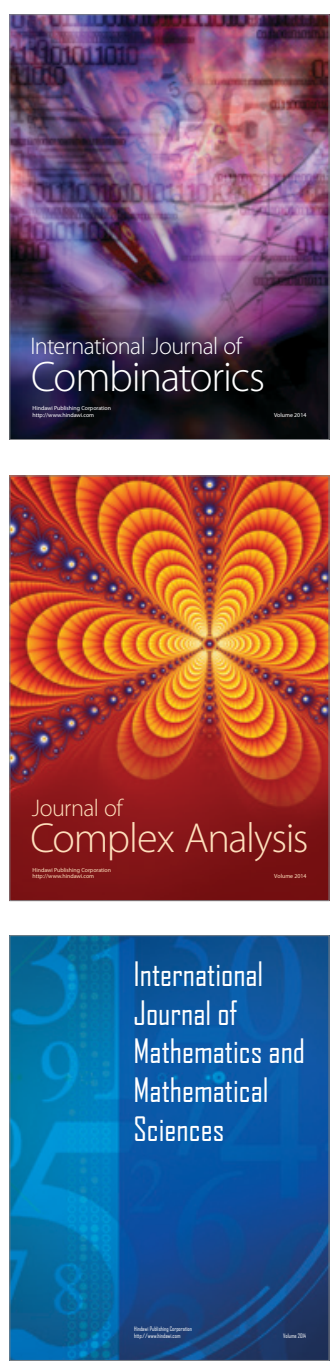
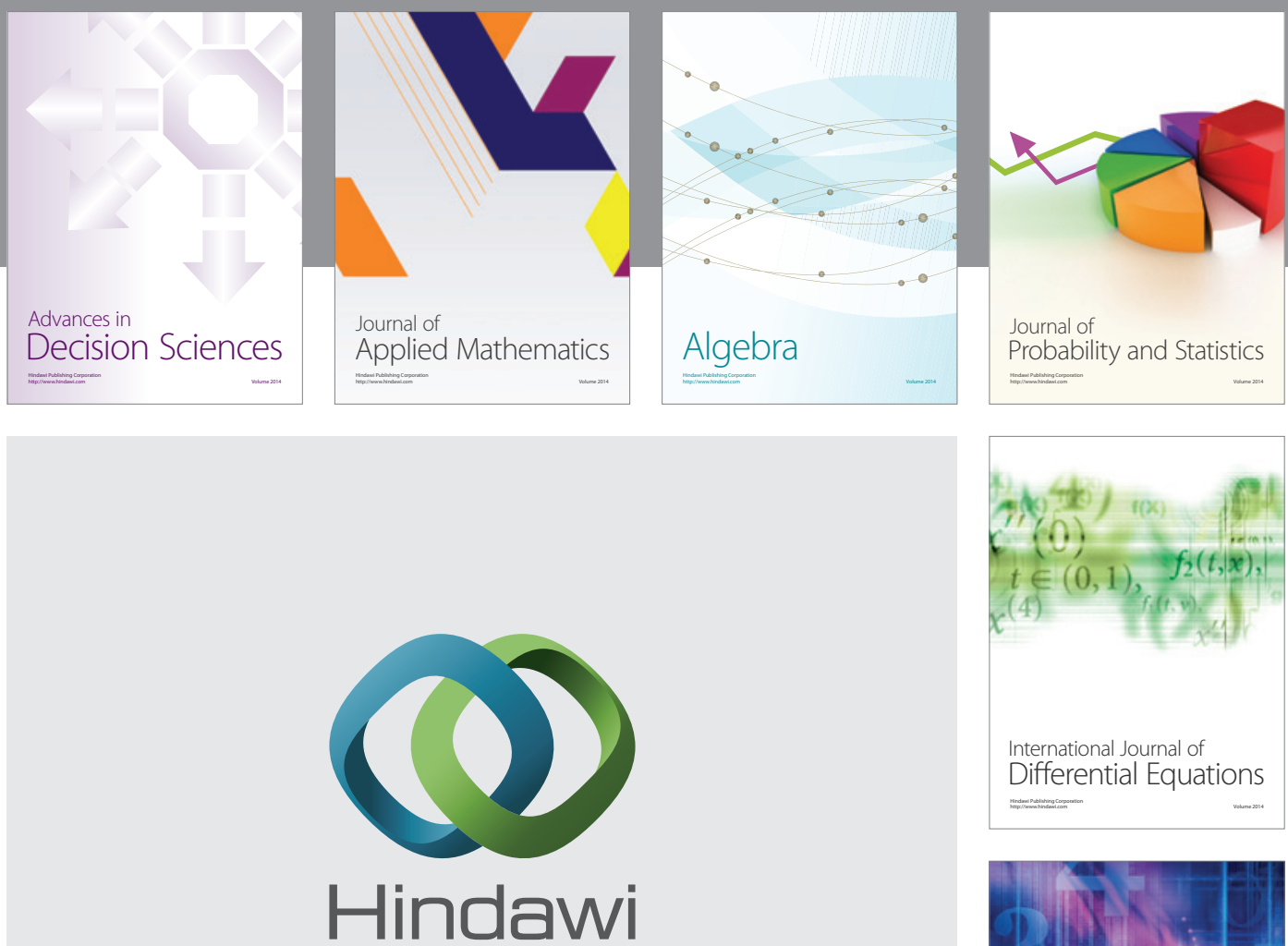

Submit your manuscripts at http://www.hindawi.com
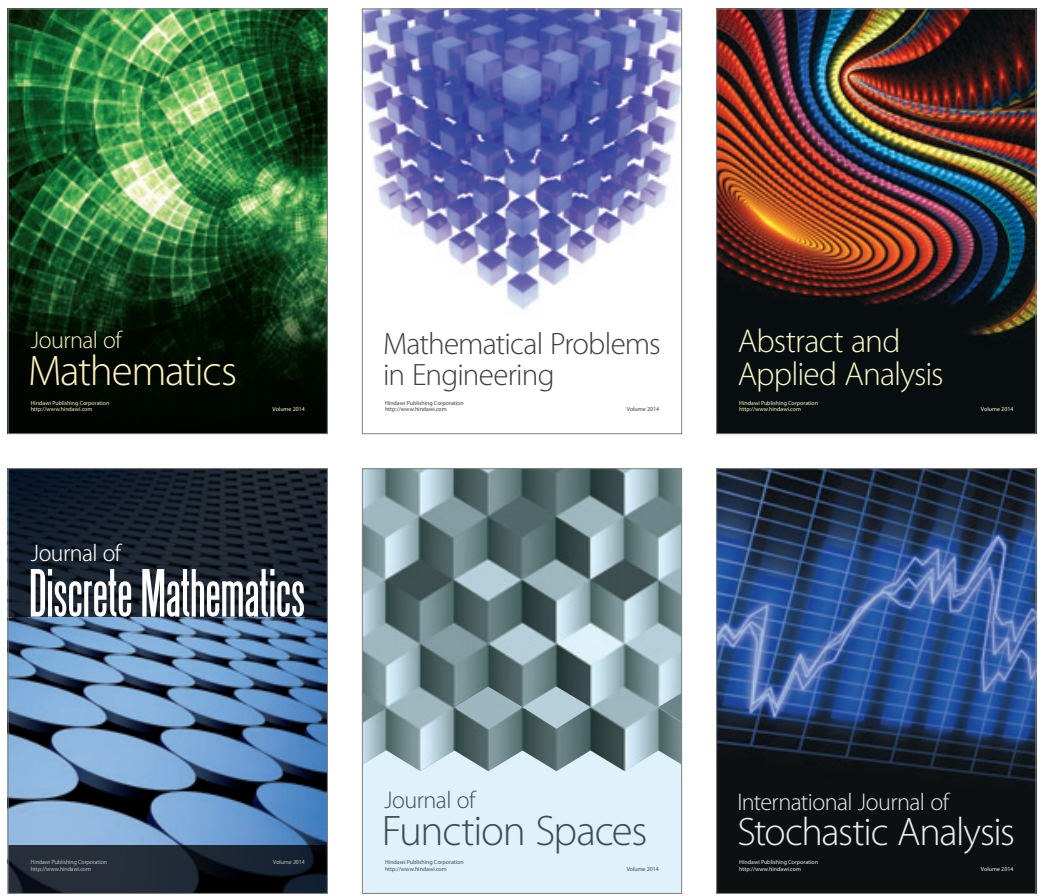

Journal of

Function Spaces

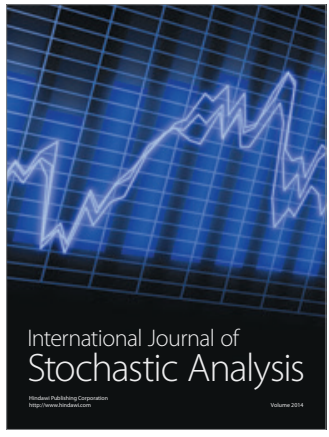

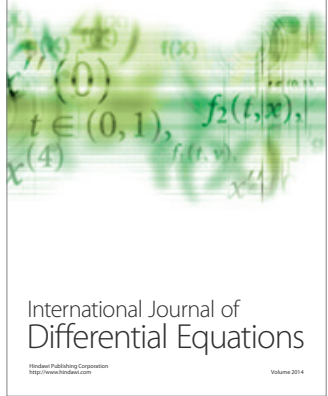
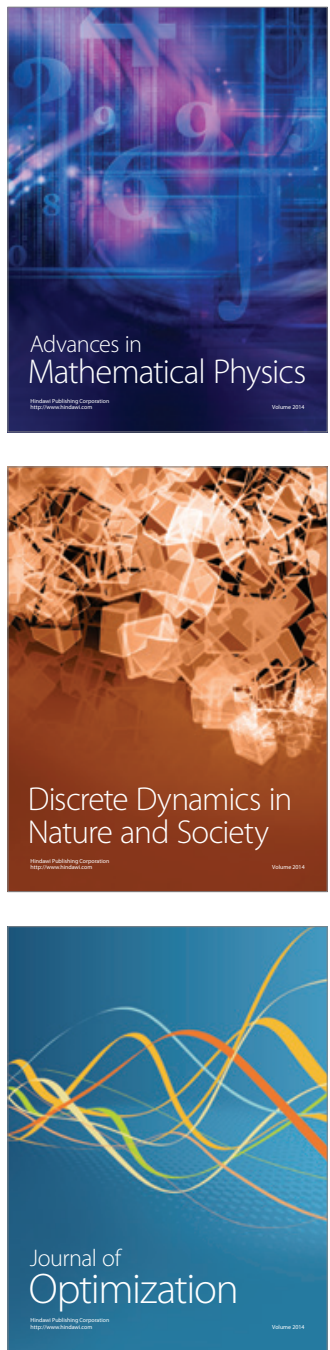